\title{
A Beam Squinted Linearly Polarised Radial Line Slot Array Antenna with Improved Return Loss Bandwidth
}

\author{
Nishat Yasmin Koli ${ }^{1}$, Muhammad U. Afzal ${ }^{1}$, Karu P. Esselle ${ }^{2}$, Raheel M. Hashmi ${ }^{1}$, and Md Zahidul Islam ${ }^{3}$ \\ ${ }^{1}$ School of Engineering, Macquarie University, Sydney, NSW, Australia \\ ${ }^{2}$ School of Electrical and Data Engineering, University of Technology Sydney, Sydney, NSW, Australia \\ ${ }^{3}$ Teleaus: Serveno Australia Pty Ltd, Sydney, NSW, Australia
}

mst-nishat-yasmin.koli@students.mq.edu.au,muhammad.afzal@mq.edu.au, karu.esselle@uts.edu.au

\begin{abstract}
This paper presents an investigation to improve the poor return loss performance of linearly polarised radial line slot array (RLSA) antenna. The problem was tackled by applying the beam squinting technique. The antenna consists of radiating surface with a circular cavity and supports outwardtravelling electromagnetic waves. The antenna is excited via a rear mounted modified disk-ended dielectric coated SMA connector. The simulation results show that the return loss performance has improved significantly over the entire frequency band with a return loss bandwidth of $52.5 \%$. The far-field result indicates that the antenna has achieved a peak directivity of $\mathbf{2 4 . 7}$ dBi at $17 \mathrm{GHz}$ with a beam squinted angle of $18^{\circ}$ and a side lobe level of $-10 \mathrm{~dB}$.
\end{abstract}

Index Terms-radial line slot array, linearly polarised, planar, low-profile, improved return loss, high gain, RLSA

\section{INTRODUCTION}

A radial-line slot-array (RLSA) antenna, is an annular slot planar antenna [1], [2] attractive for point-to-point wireless communications and receiving direct-to-home $(\mathrm{DtH})$ television (TV) programs. Geometrical simplicity, low profile and lowcost feature make the RLSA a unique choice for outdoor wireless LAN (wLAN) applications. This type of antenna belongs to the slotted waveguide arrays family, which is the most promising candidates for high gain planar antennas with the lowest conductor loss among all the other planar feeding structures such as the microstrip lines [1]. The RLSA antenna is composed of two circular parallel conductive surfaces with a waveguide formed between these two surfaces. The top conductive surface carries a distribution of radiating slots, while the bottom conductive surface acts as a ground plane. The RLSA antennas can provide either linear or circular polarisation by appropriately selecting the slot layout on the top conducting surface. Many investigation were performed to improve the radiation performance of circularly polarized RLSA antennas [1], [3]. In circularly polarized RLSA antenna the slots are spaced by a quarter guided wavelength in the radial direction producing smaller reflections at the feed point. But in linearly polarized (LP) RLSA antenna slots are arranged with a spacing of half-guided wavelength in the radial direc- tion, producing greater reflections from adjacent slot pairs that add in phase at the input feed point. Higher reflections create a very poor return loss performance in LP RLSA antenna [2], [4]. Reflection-cancelling slots and beam tilting techniques are used to improve the return loss performance in the LP-RLSA antenna.

This paper presents an investigation on linearly polarised RLSA antennas to improve the return loss performance. The aim is to develop a LP-RLSA antenna with a squinted beam that has a wide return loss bandwidth and lower side lobe levels.

\section{Design CONFIGURATION}

Fig. 1 shows the front view of the top radiating surface and cross-section view of the beam squinted linearly polarised RLSA antenna. In this arrangement, the antenna consists of two metal plates made with copper. The radial cavity formed between these plates is filled with a dielectric material and air

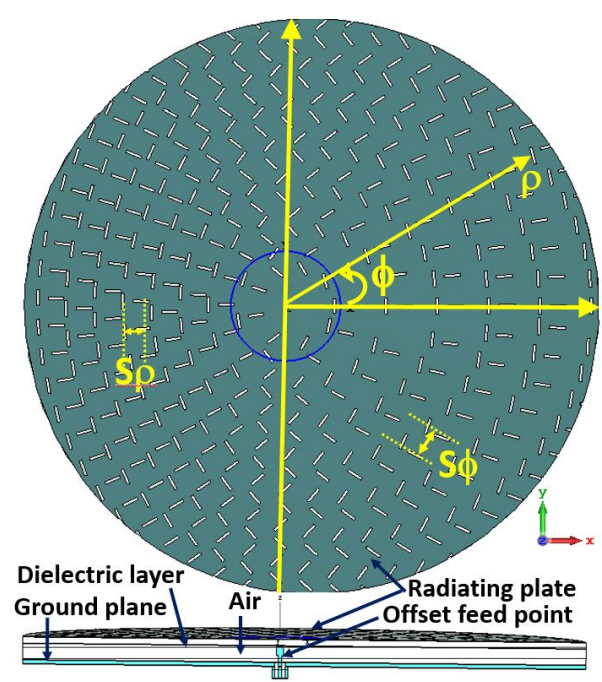

Fig. 1. Configuration of the beam squinted linearly polarised RLSA antenna. 


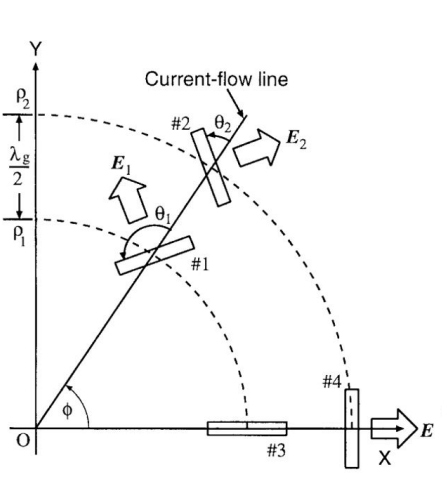

Unit radiator of LP-RLSA antenna

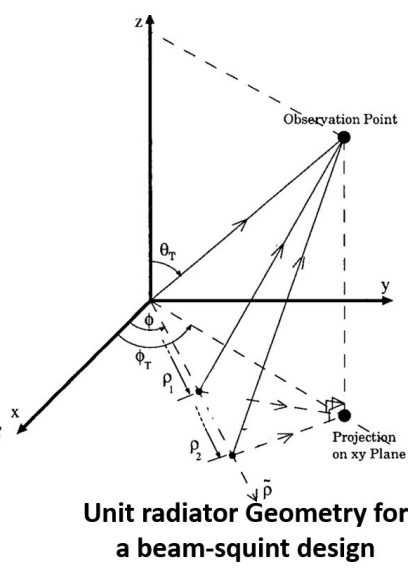

a beam-squint design
Fig. 2. Unit radiator geometry for a beam squinted LP-RLSA [4].

The power is fed to the antenna via a $50 \Omega$ coaxial to waveguide transition feed. The feed is modified with disk ended head which ensures proper matching of the radial waveguide to the coaxial transmission line. The feed converts the power from transverse electromagnetic (TEM) transmission mode to TEM waveguide mode. The energy radiated from the feed travel radially outward into the waveguide. Fig. 2 shows the geometry of the LP-RLSA antenna showing the beam squinting parameters which defines the orientation of the slots on the radiating surface [4]. The geometry shows the unit radiator condensed to a single element, and the observation point being the main beam direction. $\phi$ is the angle between the direction of propagation and the position of slot on the radiating surface. $\phi_{T}$ is the angle between the direction of propagation and the projection on XY plane. $\theta_{T}$ is the squint angle. The antenna was designed at $17 \mathrm{GHz}$ with a squinted angle of $18^{\circ}$. The unit radiators on the radiating surface are arranged in a way so that the resulting radiation contributes in phase in the desired main beam direction at $\theta_{T}, \phi_{T}$.

\section{Simulation Results}

The antenna was simulated in the time domain solver of CST Microwave Studio. Fig. 3 shows the return loss

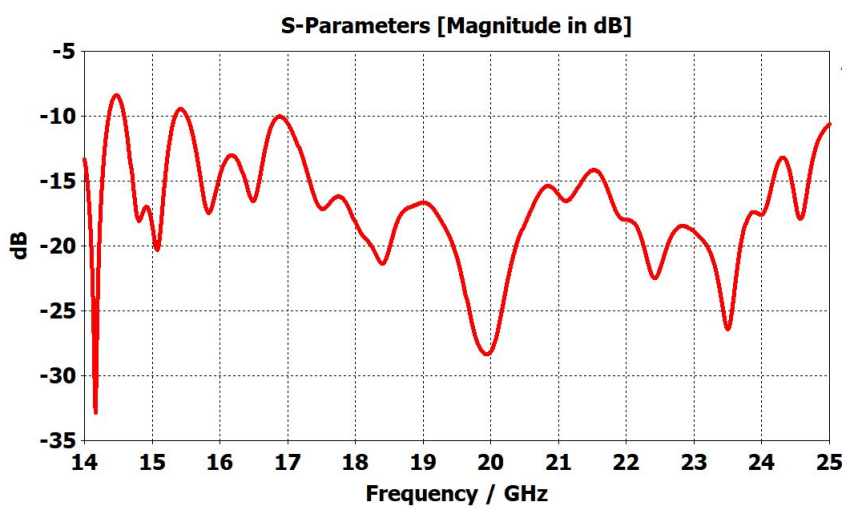

Fig. 3. Return loss magnitude, $\left|S_{11}\right|$ of the LP-RLSA antenna.

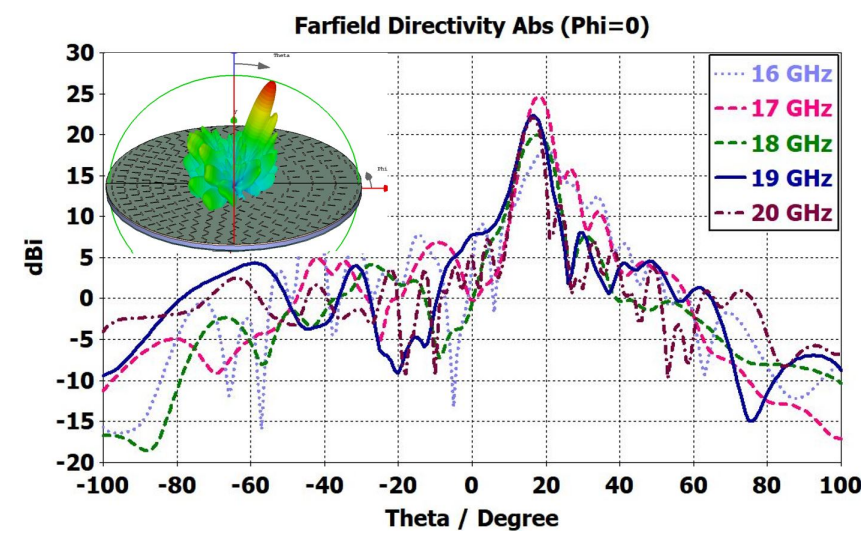

Fig. 4. Far-field directivity pattern cuts taken at five different frequencies in E-plane.

magnitude, $\left|S_{11}\right|$ of the beam squinted linearly polarised RLSA antenna. A significant improvement has been made in the return loss performance over the entire frequency band of interest. The antenna has achieved a return loss bandwidth of $52.5 \%$ from $14.6 \mathrm{GHz}$ to $25 \mathrm{GHz}$. However, within the frequency band of interest, between 15.35 and $15.5 \mathrm{GHz}$, the LP-RLSA showed a return loss magnitude of $-9.6 \mathrm{~dB}$. The farfield directivity patterns taken at five different frequencies in E-plane are plotted in Fig. 4. The antenna has achieved a peak directivity of $24.7 \mathrm{dBi}$ and a realized gain of $24.1 \mathrm{dBi}$ at the operating frequency of $17 \mathrm{GHz}$ with the main beam squinted at an angle of $18^{\circ}$. The antenna has also showed lower side lobe levels (SLL) $(>-10 \mathrm{~dB})$. The SLLs in E-plane mostly remain close to $-16 \mathrm{~dB}$ and rise up to $-10 \mathrm{~dB}$ near the lower end of the bandwidth.

\section{CONCLUSION}

In this paper we have reported preliminary results of our investigation to improve the return loss bandwidth of LPRLSA antenna using the beam squinted technique. The beam squinted of LP RLSA antenna has been demonstrated with the production of a squinted main beam pointed in an arbitrary direction. The antenna has showed a very good return loss bandwidth of $52.5 \%$ with a peak gain of $24.1 \mathrm{dBi}$. The antenna has also achieved a radiation efficiency of $95 \%$ and a total efficiency of $87.6 \%$ at $17 \mathrm{GHz}$.

\section{REFERENCES}

[1] M. Ando, K. Sakurai, N. Goto, K. Arimura, and Y. Ito, "A radial line slot antenna for $12 \mathrm{GHz}$ satellite TV reception," IEEE Transactions on Antennas and Propagation, vol. 33, no. 12, pp. 1347-1353, Dec 1985.

[2] P. W. Davis and M. E. Bialkowski, "Experimental investigations into a linearly polarized radial slot antenna for DBS TV in Australia," IEEE Transactions on Antennas and Propagation, vol. 45, no. 7, pp. 11231129, Jul 1997.

[3] M. Ando, K. Sakurai, and N. Goto, "Characteristics of a radial line slot antenna for $12 \mathrm{GHz}$ band satellite TV reception," IEEE Transactions on Antennas and Propagation, vol. 34, no. 10, pp. 1269-1272, 1986.

[4] P. W. Davis and M. E. Bialkowski, "Linearly polarized radial-line slotarray antennas with improved return-loss performance," IEEE Antennas and Propagation Magazine, vol. 41, no. 1, pp. 52-61, Feb 1999. 